\title{
Source reliability in a multiple cue probability processing task
}

\author{
PAUL J. COOK and LOWELL M. SCHIPPER \\ Bowling Green State University, Bowling Green, Ohio
}

\begin{abstract}
The reliabilities of information sources concerning two events were manipulated in a multiple cue probability processing task. Five treatments were used in which varying levels of reliability were associated with cues that were of higher or lower probability and were outlier or nonoutlier cues. Different assessments of occurrence of events showed that the reliability of a source of information influenced the subjects' decisions. A cue of high reliability increased preference for that event with which it was associated. A cue of low reliability decreased preference. The strongest preferences occurred when high reliability was associated with a high-probability cue; the weakest preferences occurred when low reliability was associated with a low-probability cue. These results were consistent in each of two replications.
\end{abstract}

Source unreliability has been studied with a variety of decision-making paradigms. In some cases, differential reliability has influenced decisions. For example, Rucci and Schipper (1978) found that semantically communicated source reliability affected predictions of future events. Levin, Ims, and Vilmain (1980) manipulated variability of test scores and grades of hypothetical students. When subjects were instructed to consider source reliability, they differentially weighted information. Uninstructed subjects did not. But other studies have shown that source reliability is not necessarily considered in decision-making (e.g., Kahneman \& Tversky, 1973; Tversky \& Kahneman, 1971, 1974).

Normative models have been developed to include estimates of observational uncertainty in the calculation of posterior probabilities (Gettys \& Willke, 1969; Schum \& DuCharme, 1971). But even when source reliability is considered, resulting decisions may not be normative. Several studies have compared decisions in a cascaded inference task with those predicted with various normative models (Snapper \& Fryback, 1971; Steiger \& Gettys, 1972; Youssef \& Peterson, 1973). These studies showed more extreme posterior probabilities (p) than those expected from the normative models.

The purpose of the present study is to examine further the effects of source reliability when the information about two events is from independent sources, as in the multiple cue probability processing (MCPP) paradigm (Jones, Schipper, \& Holzworth, 1978). This paradigm presents two sets of different estimates of $p$ values of occurrence of two events that are to be compared with each other.

The late Lowell M. Schipper had participated in all but the final draft of this article before his death in August 1984. The comments and suggestions made by Michael E. Doherty during the preparation of this paper are greatly appreciated. Requests for reprints should be sent to Paul J. Cook, Department of Psychology, Bowling Green State University, Bowling Green, OH 43403.
The subject decides which event is more likely to occur. Note that this is not the typical Bayesian task in which subjects are asked to integrate prior probabilities and likelihood ratios. In the MCPP paradigm, subjects are given differing estimates of event probabilities directly (i.e., not $\mathrm{P}(\mathrm{D} \mid \mathrm{H})$ values) and asked to integrate those probabilities. To the authors' knowledge, no appropriate normative model exists.

Jones et al. (1978), using the MCPP paradigm, showed that subjects tended to discount (i.e., ignore or reduce weightings of) discrepant information. If the reliability of the source of such discrepant information were enhanced, there might be less discounting. If the reliability of discrepant information were lower than that of other cues, discounting might be greater.

Also, in Jones et al. (1978), a strategy equivalent to averaging sets of probabilities and comparing those averages was used most frequently. On the basis of pilot data (reported in Schipper \& Doherty, 1983), we speculated that there might be a shift to other (simpler?) strategies, such as the use of absolute number of cues, the highest cue value, or the highest sum of cue values when reliability was manipulated in the paradigm or when event averages were not diagnostically useful.

\section{METHOD}

\section{Subjects}

Eighteen females and two males from undergraduate statistics classes served as paid subjects.

\section{Experimental Materials}

The subjects were presented two replications of 100 trials each. In each trial, the subjects were asked to evaluate information about two events and decide which event was more likely to occur. Two types of information were to be evaluated for each event: $p$ values (cues) of event occurrence, and the reliability of the sources of those cues.

Each trial was presented individually on an Apple II Plus computer. A vertical scale of $p$ values ranging from .20 to .80 in increments of .10 was displayed. A letter adjacent to one of the $p$ values represented a single source of information (cue) about one of the two events. Let- 
ters on the left side of the display referred to an event labeled " $D$," and those on the right, to event "J." For each event, there were two, three, or four cues. The letters $\mathrm{L}, \mathrm{M}$, and $\mathrm{H}$ were used to indicate cues and referred to low, medium, and high levels of cue reliability, respectively. An example of the display for one trial is shown in Figure 1A. There are three cues for event " $D$ "' and four cues for event "J." Two cues, .20 and .30 , are of medium reliability, and indicate $p$ values of the occurrence of event " $D$." The third cue for " $D$ "' is highly reliable and specifies a $p$ of .70 that the event will occur. All cues for " $\mathrm{J}$ "' are of medium reliability.

In order to assess the effect of varying cue reliabilites, five treatments were selected. The objective was to compare subjects' decisions (event selections) in a baseline treatment [in which all cues were of equal (medium) reliability] with decisions in treatments with the same distribution of $p$ values but in which one corresponding cue was changed to high or low reliability. Twenty patterns of cues ( $p$ values) were selected, and each pattern was repeated in the five treatments, with only the reliabilities of the cues varying across treatments. The rationale for the cue patterns will be discussed first, followed by a description of each treatment.

Cue patterns that would be diagnostic in isolating various selection strategies when all of the cues were of equal (medium) reliability were selected. Combinations that presented trivial problems (e.g., .50 and .80 for event ' $D$ "' paired with .20 for event " $J$ "') were, of course, not used. Jones et al. (1978) suggested four simple strategies readily available to judges: an averaging strategy (prediction of the event with the larger cue average); an additive strategy (prediction of the event with the larger sum of the cue values); a largest-cue strategy (prediction of the event with the largest single cue value); and a most-cues strategy (prediction of the event with the largest number of cues). In this study, cue patterns were selected such that a most-cues, a largest-cue, or an additive strategy would result in selection of the event with an outlier cue (defined below), when all cues were of medium reliability, about $50 \%$ of the time. Our pilot data indicated that these, or some strategy other than averaging, were most likely used. A $50 \%$ selection rate in the baseline treatment allowed selection rates to increase or decrease in the treatments with differing reliability.

Another consideration in selecting cue patterns involved the use of discrepant information. Manipulating the reliability of the source of discrepant information might influence the discounting noted in Jones et al. (1978). Thus, in all trials, one event contained an outlier cue. An outlier is defined as a single cue that specifies a $p$ value at least .30 distant from the nearest cue in a cluster of at least two other predictors of that event (e.g., in the set of cues $.20, .60$, and .70 , the outlier is .20).

\begin{tabular}{|c|c|c|c|c|c|c|c|c|c|c|c|}
\hline \multicolumn{3}{|c|}{ (A) Sample Item } & \multicolumn{9}{|c|}{ (B) Cue Patterns } \\
\hline \multirow{2}{*}{$\frac{\text { Event }}{\underline{0}}$} & & \multirow{2}{*}{$\frac{\text { Event }}{\underline{J}}$} & \multicolumn{2}{|c|}{ Pattern } & \multicolumn{3}{|c|}{ Outlier Event Cues } & \multicolumn{4}{|c|}{ Non-Outlier Event Cues } \\
\hline & & & 1 & & .20 & .60 & .70 & & .40 & .50 & .60 \\
\hline & & & 2 & & .20 & .60 & .70 & & & .50 & .60 \\
\hline \multirow{5}{*}{$\begin{array}{l}M \\
M\end{array}$} & .20 & & 3 & & .20 & .60 & .70 & & .40 & .60 & .80 \\
\hline & .30 & m & 4 & & .20 & .60 & .70 & & & .60 & .70 \\
\hline & .40 & M & 5 & & .20 & .60 & .70 & & .60 & .70 & .80 \\
\hline & .50 & M & 6 & & .20 & .30 & .70 & & .30 & .40 & .50 \\
\hline & .60 & M & 7 & & .20 & .30 & .70 & & & .40 & .50 \\
\hline \multirow[t]{13}{*}{ H } & .70 & & 8 & & .20 & .30 & .70 & & .30 & .50 & .70 \\
\hline & .80 & & 9 & & .20 & .30 & .70 & & & .50 & .60 \\
\hline & & & 10 & & .20 & .30 & .70 & & .40 & .60 & .80 \\
\hline & & & 11 & .20 & .30 & .40 & .70 & & .40 & .50 & .60 \\
\hline & & & 12 & .20 & .30 & .40 & .70 & .40 & .50 & .60 & .70 \\
\hline & & & 13 & .20 & .30 & .40 & .70 & & .50 & .60 & .70 \\
\hline & & & 14 & .20 & .30 & .40 & .70 & .50 & .60 & .70 & .80 \\
\hline & & & 15 & .20 & .30 & .40 & .70 & & .60 & .70 & .80 \\
\hline & & & 16 & .20 & .50 & .60 & .70 & & .40 & .50 & .60 \\
\hline & & & 17 & .20 & .50 & .60 & .70 & .40 & .50 & .60 & .70 \\
\hline & & & 18 & .20 & .50 & .60 & .70 & & .50 & .60 & .70 \\
\hline & & & 19 & .20 & .50 & .60 & .70 & .50 & .60 & .70 & .80 \\
\hline & & & 20 & .20 & .50 & .60 & .70 & & .60 & .70 & .80 \\
\hline
\end{tabular}

Figure 1. (A) Sample MCPP task trial. (B) Pattern of cue values selected for the five treatments.
The 20 cue patterns were selected such that there were three or four cues in the arrays with the outlier cue and two, three, or four cues in the nonoutlier cue arrays. In half of the patterns, the outlier had a high probability, and in the other half it had a low probability (see Figure 1B).

Across the five treatments, all cues within the nonoutlier array were of medium reliability, and only cues for the outlier events varied in reliability, even though this arrangement confounded differential reliability with the outlier versus the nonoutlier cue events.

In Treatment 1 (T1), the baseline treatment, all outlier events cues were of medium reliability; in $\mathrm{T} 2$, the outlier cue was of high reliability; in T3, the outlier cue was of low reliability; and in T4, one of the nonoutlier cues was of high reliability (for events with a low $\mathrm{p}$ outlier, the second highest nonoutlier cue was of high reliability; for events with a high $\mathrm{p}$ outlier, the second lowest nonoutlier cue was of high reliability). In T5, one of the nonoutlier cues was of low reliability (for events with a low p outlier, the second highest nonoutlier cue was of low reliability; for events with a high p outlier, the second lowest nonoutlier cue was of low reliability).

Thus, each of the 20 cue patterns described in Figure 1B occurred in each of the five treatments, for a total of 100 trials. Order of trials was randomized separately for each subject, and the event with the outlier cue appeared as event " $D$ " in half of the trials and as event " $J$ ", in the other half. The entire set was presented twice to each subject in different random orders for each replication to measure the consistency of the subjects' selections.

\section{Procedure}

The subjects were run individually. At the outset, they were asked what the term "reliability" meant to them, questioned to clarify their definitions, and asked to describe the terms "high, medium, and low reliability."' Responses were tape-recorded. (Due to space constraints, these data will not be presented.)

Three trials, none of which were the same as those in the experiment proper, were used to demonstrate the task. On each trial, the subjects were to decide which of the two events was more likely and to press the appropriate key, D or J, on the computer to record the decision. The initial session of 100 trials was followed by a short break followed by the second 100 trials. Next, tape recordings of the subjects' descriptions of their selection strategies were made. Dependent variables were the frequency of selection of events with the outlier cue and reaction times.

\section{RESULTS}

The mean frequencies of outlier event selections for each treatment across both replications are shown in Table 1 . The possible ranges of frequencies are 0 to 20 for the combined set of trials and 0 to 10 for each replication (recall that the 20 trials in each treatment had 10 events with high and 10 events with low $p$ value outlier cues). A test for compound symmetry of the variance-covariance matrices showed the univariate assumption not to be tenable for either low or high outlier trials. Thus, Hotelling's $\mathrm{T}^{2}$ statistic was used as an overall test for differences among the means of the five treatments. These $T^{2} s$ all showed reliable differences among all treatments, collectively, for each replication and for the combined frequency data. Individual pairwise comparisons of T1 (the control) with each of the other four treatments are shown in Table 1.

Similar analyses of mean reaction times for the various treatments were made. There were no reliably different means overall, and those results are not presented.

Because each subject was presented with the same 100 trials twice (in the two successive 100-trial blocks), subject consistency can be expressed as the number of times 
Table 1

Mean Frequency Outlier Event Selection

\begin{tabular}{|c|c|c|c|c|c|}
\hline & \multicolumn{5}{|c|}{ Treatment $^{\mathrm{a}}$} \\
\hline & 1 & 2 & 3 & 4 & 5 \\
\hline & Base Med Rel & Outlier Hi Rel & Outlier Lo Rel & Cluster Hi Rel & Cluster Lo Rel \\
\hline \multicolumn{6}{|c|}{ Frequency of Low Outlier Trials } \\
\hline Mean Repl. 1 & 3.90 & 5.90 & 3.45 & 8.60 & 1.10 \\
\hline Mean Repl. 2 & 4.60 & 5.95 & 3.65 & 8.55 & 1.45 \\
\hline Mean Comb. & 8.50 & 11.85 & 7.10 & 17.15 & 2.55 \\
\hline SD & 3.47 & 4.40 & 2.45 & 2.80 & 3.27 \\
\hline$t_{(} T_{1}$ vs. $\left.T_{i}\right)$ & & $-4.78 *$ & 1.75 & $-8.49 *$ & $8.53 *$ \\
\hline \multicolumn{6}{|c|}{ Frequency of High Outlier Trials } \\
\hline Mean Repl. 1 & 2.40 & 7.00 & 0.55 & 4.75 & 1.00 \\
\hline Mean Repl. 2 & 3.00 & 7.85 & 0.70 & 5.35 & 1.55 \\
\hline Mean Comb. & 5.40 & 14.85 & 1.25 & 10.10 & 2.55 \\
\hline SD & 3.59 & 4.63 & 2.15 & 4.90 & 2.21 \\
\hline$t_{\left(T_{1} \text { vs. } T_{i}\right)}$ & & $-7.59 *$ & $6.31 *$ & $-5.36^{*}$ & $4.62 *$ \\
\hline
\end{tabular}

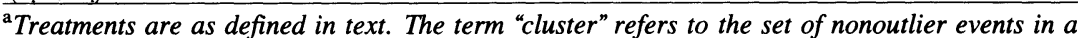
trial in Treatments 4 and 5. " "Comb." refers to the two replications combined. Each Mean Comb. is based on 20 trials. $\quad * p<.001$.

the same events were selected in corresponding trials of both replications. This mean consistency was 86.7 (SD $=3.39$, range $=78$ to 93 ). Another assessment of consistency can be made by comparing respective paired entries in Table 1 for Replications 1 and 2.

Individually, the majority of subjects produced a pattern of frequencies across treatments similar to the pattern of overall means.

For events with low outlier cues, (1) T2 outlier event selection was greater than $\mathrm{T} 1$ outlier event selection for 16 subjects and was tied for 2 subjects, (2) T3 was less than T1 for 12 subjects and was tied for 3, (3) T4 was greater than $\mathrm{T} 1$ for all 20 subjects, and (4) T5 was less than $\mathrm{T} 1$ for all 20 subjects.

For events with high outlier cues, (1) T2 was greater than T1 for 19 subjects, (2) T3 was less than T1 for 17 subjects and was tied for 1, (3) T4 was greater than T1 for 18 subjects and was tied for 1 , and (4) T5 was less than $\mathrm{T} 1$ for 17 subjects and was tied for 1 .

\section{DISCUSSION}

Differential reliability of information, communicated semantically, obviously influenced decision behavior in this task. Specifically, for events in which the reliability of a single cue was enhanced, selection of those events increased markedly over the frequencies in the treatment in which all cues were of medium reliability (means of T2 and T4 were higher than the respective means of T1). The highest outlier event selections occurred when a high-probability cue (.60 or .70) was of high reliability (T4 for low outlier events and T2 for high outlier events).

When the reliability of any single cue was reduced, outlier event selections decreased (means of T3 and T5 for both high and low outliers were lower than the respective means of $\mathrm{T} 1$ ). The lowest outlier event selections occurred when a high-probability cue was of low reliability (T5 for low outlier events and T3 for high outlier events).

These results are somewhat inconsistent with the speculated effects of enhanced and diminished reliability for outliers. The treatments that included a highly reliable cue consistently enhanced the perceived probability of occurrence of that event to which that cue was related. The treatments with a low-reliability cue showed decreased selection of the event with which that cue was related. In other words, discounting of outliers and appropriate weighted averaging did not occur consistently. It appeared as though the subjects used a highly reliable cue for the to- be-predicted event as an enhancer of the likelihood of that event even if that cue had a $\mathrm{p}$ value of less than .50 . The appropriate weighting and use of the probabilistic predictors was inconsistent across high and low $p$ values, given a normative interpretation of probability.

Additionally, it appears that the averaging strategy that predominated in Jones et al. (1978) and in Schipper and Doherty (1983) was not used by most subjects in this study. It may be that a low-probability cue of high or medium reliability was considered as simply one additional bit of evidence in some form of additive or most-cues strategy, rather than averaged as a low-probability cue providing evidence against the occurrence of the event.

Support for the use of an additive, a most-cues, or a largest-cue strategy can be found by examining data for individual trials in the baseline treatment. For low outlier trials, there was strongest preference for outlier events with more cues, the largest cue, and the highest total (Cue Patterns 2 and 16). Next highest preference occurred when the largest cues were tied in value but the outlier event had more cues and a higher total (Cue Patterns 4 and 18). Fewest selections occurred when the nonoutlier event had the largest cue, higher total, or higher average (Cue Patterns 3,5 , and 19). None of this clearly establishes the predominance of a particular strategy, but the results do argue against a simple averaging strategy. Had only an averaging strategy been used, outlier event selections would have been zero for all low outlier cue patterns except cue patterns 1 and 16 (in which the cue average was the same for both events).

There is also some support for use of strategies other than simple averaging to be found in T1 with high outlier events. Greatest preferences were shown with outlier events having more cues, the largest cue, and the higher total (Cue Patterns 7, 9, and 11). The fewest outlier event selections occurred for Cue Patterns 8,10,12, and 14, in which the non-outlier event had the higher total, largest cue, or higher average.

In sum, this study showed that people do consider the reliability of a source of information when they make decisions in a MCPP task (although somewhat illogically, in that they use reliable, low-probability cues as evidence for an event). The results of individual trials were examined to try to isolate decision strategies that would account for the pattern of results. An averaging strategy, either unweighted or weighted by reliability information, was not the most frequently used approach here, but consistent use of other strategies could not be confirmed.

\section{REFERENCES}

Gettys, C. F., \& Willke, T. A. (1969). The application of Bayes's theorem when the true data state is uncertain. Organizational Behavior and Human Performance, 4, 125-141.

Jones, D. P., SCHIPPER, L. M., \& HolzWorth, R. J. (1978). Effects 
of amount of information on decision strategies. Journal of General Psychology, 98, 281-294.

KAHNEMAN, D., \& TVERSKY, A. (1973). On the psychology of prediction. Psychological Review, 80, 237-251.

LeVIN, I. P., IMS, J. R., \& Vilmain, J. A. (1980). Information variability and reliability effects in evaluating student performance. Journal of Experimental Psychology, 72, 355-361.

RUCCI, A. J., \& SCHIPPER, L. M. (1978, November). Estimation of likelihoods of events as a function of impact and reliability of predictors. Paper presented at the annual meeting of The Psychonomic Society, San Antonio.

SCHIPPER, L. M., \& Doherty, M. E. (1983). Decision making and information processing under various uncertainty conditions (AFHRL Tech. Rep. No. 83-19). San Antonio: Brooks Air Force Base, HQ Air Force Human Resources Laboratory.
Schum, D. A., \& DuCharme, W. M. (1971). Comments on the relationship between the impact and the reliability of evidence. Organizational Behavior and Human Performance, 6, 111-131.

SNAPPER, K. J., \& FrybaCK, D. G. (1971). Inferences based on unreliable reports. Journal of Experimental Psychology, 87, 401-404.

Steiger, J. H., \& GetTys, C. F. (1972). Best guess errors in multistage inference. Journal of Experimental Psychology, 92, 1-7.

Tversky, A., \& Kahneman, D. (1971). Belief in the law of small numbers. Psychological Bulletin, 2, 105-110.

TVERSKy, A., \& KahNEMAN, D. (1974). Judgment under uncertainty: Heuristics and biases. Science, 185, 1124-1131.

Youssef, Z. I., \& Peterson, C. R. (1973). Intuitive cascaded inference. Organizational Behavior and Human Performance, 10, 349-358.

(Manuscript received for publication October 29, 1984.) 